\title{
Efficacy of Two-Dimensional Perfusion Angiography for Evaluations after Infrapopliteal Bypass Surgery for Critical Limb Ischemia
}

\author{
Fumiya Yoneyama, MD, ${ }^{1}$ Motoo Osaka, MD, PhD, ${ }^{1}$ Fujio Sato, MD, PhD, ${ }^{2}$ \\ Hiroaki Sakamoto, MD, PhD, ${ }^{1}$ and Yuji Hiramatsu, MD, PhD ${ }^{1}$
}

Two-dimensional perfusion angiography (2DPA) is utilized in hybrid operating rooms. 2DPA produces color map images and functional parameters to provide more robust visual and quantitative evaluations than conventional angiography. Its efficacy was suggested in five patients following bypass surgery; unexpected results were obtained in one patient, leading to a decision to perform surgical re-anastomosis. Furthermore, we found that the general anesthesia eliminates body movements that tend to disrupt 2DPA results. 2DPA was more useful during surgical revascularization than conventional angiography and provided more detailed information.

Keywords: 2D perfusion angiography, infrapopliteal bypass surgery, critical limb ischemia

\section{Introduction}

Tools such as the ankle-brachial index (ABI), skin perfusion pressure (SPP), and conventional angiography have been postoperatively used to evaluate peripheral blood perfusion after revascularization. ${ }^{1)}$ Recently, two-dimensional perfusion angiography (2DPA) has been used to evaluate peripheral perfusion in the foot after endovascu-

${ }^{1}$ Department of Cardiovascular Surgery, University of Tsukuba Hospital, Tsukuba, Ibaraki, Japan

${ }^{2}$ Department of Cardiovascular Surgery, Tsukuba Medical Center Hospital, Tsukuba, Ibaraki, Japan

Received: December 27, 2017; Accepted: February 22, 2018 Corresponding author: Fumiya Yoneyama, MD. Department of Cardiovascular Surgery, University of Tsukuba Hospital, 1-3-1 Amakubo, Tsukuba, Ibaraki 305-8558, Japan

Tel: +81-298-51-3511, Fax: +81-298-53-3097

E-mail: fumiya.y@hotmail.co.jp

(cc) BY-NC-SA (9)2018 The Editorial Committee of Annals of Vascular Diseases. This article is distributed under the terms of the Creative Commons Attribution License, which permits use, distribution, and reproduction in any medium, provided the credit of the original work, a link to the license, and indication of any change are properly given, and the original work is not used for commercial purposes. Remixed or transformed contributions must be distributed under the same license as the original. lar therapy $(\mathrm{EVT})^{2-5}$ ) for peripheral artery disease (PAD). However, few studies have evaluated its usefulness for assessing surgical revascularization. In this case series, we used 2DPA evaluation after infrapopliteal bypass anastomosis in hybrid operating rooms. We discuss the efficacy of 2DPA compared with conventional angiography for evaluating surgical revascularization.

\section{Patients and Methods}

This case series includes five patients who underwent infrapopliteal bypass surgery for critical limb ischemia (CLI) at our institute, all of whom were intraoperatively evaluated using 2DPA. The patients' characteristics are shown in Table 1. Four of the five patients had diabetes mellitus and required hemodialysis.

\section{Operative procedures}

In the operation room, $10 \mathrm{cc}$ of iodinated contrast material (iopamidol, $300 \mathrm{mg}$ iodine/mL; Bayer, Osaka, Japan) was injected at two time points (before and after surgical revascularization) using a continuous injection system, at a rate of $3 \mathrm{cc} / \mathrm{s}$. We captured digital subtraction angiography images of the foot using a Philips Allura Xper FD 20 system (Philips Healthcare, Best, The Netherlands). The pre- (Figs. 1A and 1B) and post-revascularization color map images (Figs. 1C and 1D) were generated using 2D perfusion software on a Philips workstation (Interventional Workspot R1.1 with 2D Perfusion R1; Philips Healthcare). These color map images use different colors to demonstrate perfusion areas and blood stream velocities.

\section{Functional parameters of 2DPA}

To assess foot perfusion, we manually drew a wide region of interest (ROI) on the foot perfusion image, as calculated from the digital subtraction angiography. We then obtained a time-to-density curve from this 2DPA ROI, on which time duration ( $\mathrm{x}$-axis) and contrast density ( $\mathrm{y}$-axis) were plotted (Figs. 1E and 1F). The functional parameters were automatically calculated from this curve during 
Table 1 Patient characteristics

\begin{tabular}{cccccccccc}
\hline Case & Age (years), sex & Rutherford & DM & HD & Bypass procedure & Pre-ABI & Post-ABI & $\begin{array}{c}\text { Pre-SPP } \\
\text { (dorsal/plantar) }\end{array}$ & $\begin{array}{c}\text { Post-SPP } \\
\text { (dorsal/plantar) }\end{array}$ \\
\hline 1 & 49, M & 6 & + & + & Rt. Pop. A(AK)-DPA & 1.17 & 1.19 & $39 / 48$ & $57 / 69$ \\
2 & $64, \mathrm{M}$ & 5 & - & + & Rt. FA-Pop. A(BK) & ND & 0.88 & $11 /$ ND & $50 / 35$ \\
3 & $68, \mathrm{M}$ & 6 & + & + & Rt. FA-Pop. A(BK)-DPA & ND & 1.08 & $35 / 25$ & $56 / 90$ \\
4 & $76, \mathrm{~F}$ & 6 & + & + & Lt. Pop. A(AK)-PTA & 0.50 & 0.85 & $25 / 60$ & $68 / 64$ \\
5 & $77, \mathrm{M}$ & 5 & + & - & Lt. FA-Pop. A(BK) & 0.87 & 1.17 & ND/30 & $32 / 55$ \\
\hline
\end{tabular}

ABI: ankle-brachial index; AK: above knee; BK: below knee; DM: diabetes mellitus; DPA: dorsalis pedis artery; FA: femoral artery; HD: hemodialysis; Lt.: left; ND: not detected; Pop. A: popliteal artery; Post: post-revascularization; Pre: pre-revascularization; PTA: posterior tibial artery; Rt.: right; Rutherford: Rutherford classification; SPP: skin perfusion pressure
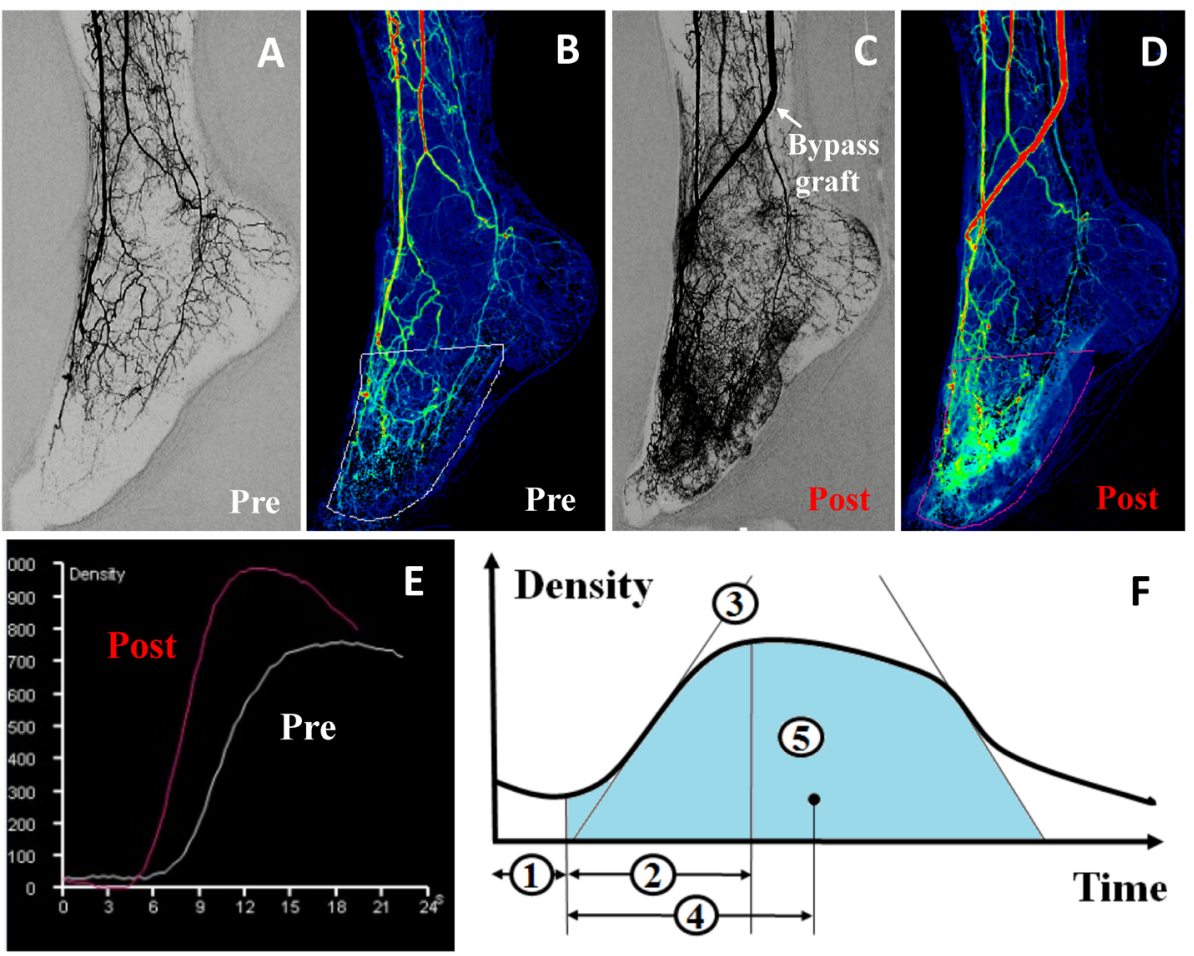

Fig. 1 (A), (B), (C), (D): Pre- and post-revascularization two-dimensional perfusion angiography images collected during identical time frames. Peripheral blood perfusion remarkably improved after revascularization. (E), (F): Pre-revascularization and post-revascularization time-density curves. Pre: pre-revascularization; Post: postrevascularization. (1) arrival time, (2) time-to-peak, (3) wash-in rate, (4) mean transit time, (5) area under the curve.

surgery. The parameters were defined and interpreted as follows: arrival time indicated the initial time to contrast detection or blood perfusion, mainly via the bypass graft after injection. Time-to-peak indicated the interval from the arrival time to the maximum contrast density. Wash-in rate was the contrast or blood flow velocity, mainly via the bypass graft. Mean transit time indicated the average time required for contrast, or perfused blood, to pass through the tissue. Area under the curve (AUC) was the estimated total tissue perfusion. The values for all functional parameters are expressed as means \pm standard errors of the means.

\section{Results}

2DPA color map images, from all five patients, indicated more rapid and widespread blood flow, thus confirming improved peripheral perfusion. We also observed improvements in several functional parameters (preoperative vs. postoperative) including arrival time, $10.7 \pm 0.9 \mathrm{~s}$ vs. $8.4 \pm 1.3 \mathrm{~s}$; time-to-peak, $8.8 \pm 1.1 \mathrm{~s}$ vs. $7.0 \pm 1.1 \mathrm{~s}$; and wash-in rate, $15.1 \pm 5.7$ vs. $25.7 \pm 8.9$ (Table 2 ).

In one patient (\#5), the 2DPA AUC decreased after revascularization, even though conventional angiography did not reveal any problems with the bypass anastomosis 
(Fig. 2). Because the AUC increased in the four other cases, we suspected anastomotic stenosis and performed a surgical distal site re-anastomosis, leading to an improvement in the AUC. Therefore, all five patients ultimately achieved improvement in the AUC from 7,491.0 $\pm 4,002.1$ preoperatively to $12,400.0 \pm 4,809.4$ postoperatively.

At one-year follow-up, all five patients experienced postoperative improvements in the ABI and SPP (Table 1). Two patients (\#2 and \#5), experienced postoperative ulcer healing, whereas three patients with gangrene (\#1, \#3, and \#4) eventually underwent skin grafting procedures for limb preservation.

\section{Discussion}

2DPA is frequently used to evaluate postoperative cerebral perfusion after carotid endarterectomy or carotid artery stenting, for carotid artery stenosis. ${ }^{6}$ A previous study found that 2DPA and conventional angiography deliver equivalent contrast and radiation doses to patients. ${ }^{1)}$ However, these modalities differ in some aspects. While conventional angiography indicates graft patency, anastomosis morphology, and distal run-off quality, 2DPA provides the surgeon with more detailed information, includ-

Table 2 Functional parameter data collected pre- and postrevascularization

\begin{tabular}{lcc}
\hline & Pre & Post \\
\hline Arrival time (s) & $10.7 \pm 0.9$ & $8.4 \pm 1.3$ \\
Time-to-peak (s) & $8.2 \pm 1.1$ & $7.3 \pm 1.1$ \\
Wash-in rate & $15.2 \pm 5.7$ & $25.7 \pm 8.9$ \\
Area under the curve & $7,491.0 \pm 4,002.1$ & $12,400.8 \pm 4,809.4$ \\
Mean transit time (s) & $7.9 \pm 1.1$ & $8.0 \pm 1.2$ \\
\hline
\end{tabular}

Pre: pre-revascularization; Post: post-revascularization ing color map images that depict the peripheral perfusion area and velocity and quantitative functional parameters indicative of peripheral perfusion "quality."

Recent studies described the efficacy of 2DPA as a postinterventional evaluation method in patients who underwent EVT for PAD. ${ }^{2-5)}$ Jens et al. suggested that improved AUC indicated more rapid ulcer healing, ${ }^{3)}$ and Hinrichs et al. reported a correlation between time-to-peak and post-interventional ABI $\left.(r=-0.53, p=0.0081) .{ }^{4}\right)$ However, these studies discussed only the earlier stages of PAD, rather than patients with deteriorated CLI (e.g., Rutherford 5 to 6) who would likely require more extensive peripheral perfusion. For patients like these, it is imperative to ensure sufficient perfusion. Furthermore, previous studies excluded some patients because of leg motion during EVT, since even trivial motion disrupts 2DPA results. In contrast, patients who are receiving general anesthesia during surgery have no detectable movement during surgical revascularization and are therefore well-suited for 2DPA. Although epidural anesthesia is another option, the epidural hematoma under using anticoagulation (heparin) during operation should be considered. We believe it is possible to obtain more precise results with 2DPA, compared with EVT, during surgical revascularization procedures.

The functional parameters of 2DPA allow for intraoperative assessment of quantitative peripheral perfusion data. Unexpected functional parameter readings, such as we experienced, can suggest failure of the bypass graft or anastomosis. In our case, these findings led us to perform re-anastomosis in one patient. Thus, 2DPA allows for intraoperative modifications to bypass planning.

Our case series has several limitations. Only five patients were included, and we did not enroll a control

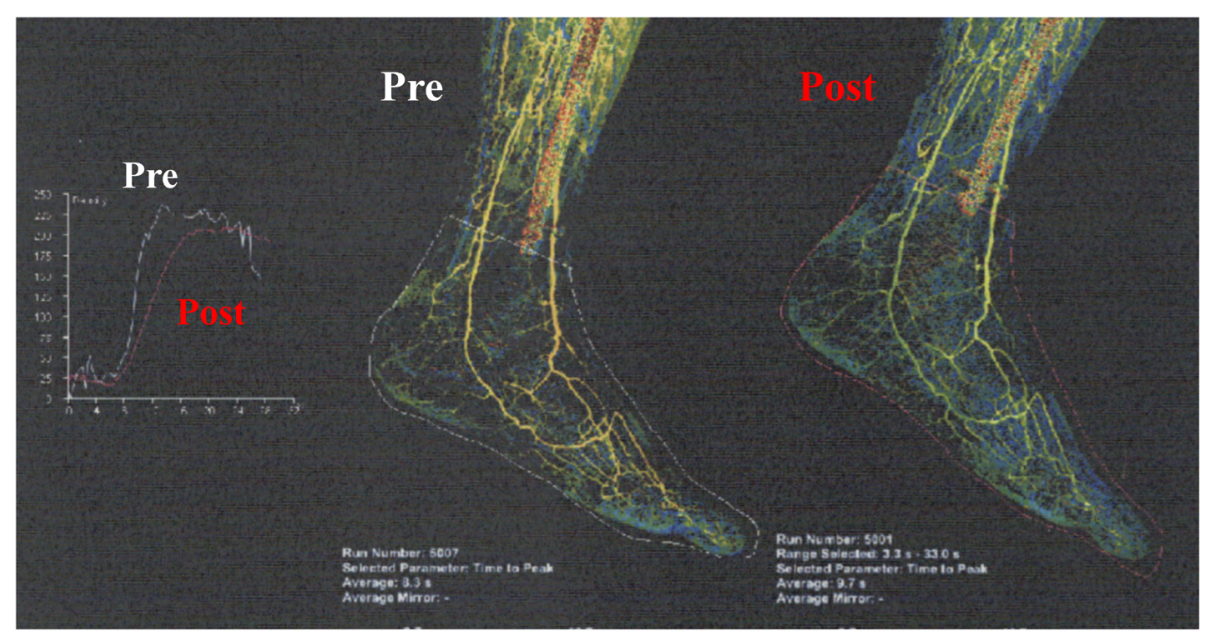

Fig. 2 Two-dimensional perfusion angiography images and the time-to-density curve in unexpected case (\#5). Peripheral blood perfusion deteriorated after revascularization. Pre: pre-revascularization; Post: post-revascularization 
group, which limited our ability to extrapolate our findings to a larger population. Additionally, 2DPA results might be affected by an arteriovenous shunt or peripheral tissue vasospasm. Renal toxicity and exacerbation associated with the use of contrast agents and general anesthesia should be considered. Furthermore, cost-effectiveness is unclear at this moment. Despite these limitations, we believe that 2DPA exhibits great potential as a technique for evaluating surgical revascularization. It is necessary to disclose the relationships between each functional parameter and the corresponding clinical findings after revascularization. Further studies should be conducted to validate the efficacy of 2DPA on peripheral tissue oxygenation, graft patency rates, and clinical outcomes.

\section{Conclusion}

2DPA produce color map images and functional parameter data, which enhance visual and quantitative evaluations. These additional data can allow the surgeon to make insightful, or unexpected, intraoperative decisions. Accordingly, 2DPA might be a useful measure of bypass surgery endpoints.

\section{Disclosure Statement}

The authors acknowledge no conflict of interest in the submission.

\section{Author Contributions}

Study conception: FY, MO, FS, YH
Data collection: FY

Analysis: FY

Investigation: FY, MO, FS

Writing: FY

Critical review and revision: all authors

Final approval of the article: all authors

Accountability for all aspects of the work: all authors

\section{References}

1) Iida O, Nakamura M, Yamauchi Y, et al. 3-year outcomes of the OLIVE registry, a prospective multicenter study of patients with critical limb ischemia: a prospective, multi-center, three-year follow-up study on endovascular treatment for infra-inguinal vessel in patients with critical limb ischemia. JACC Cardiovasc Interv 2015; 8: 1493-502.

2) Manzi M, van den Berg JC. 2D perfusion angiography: a useful tool for CLI treatment. Endovasc Today 2015; 14: 76-9.

3) Jens S, Marquering HA, Koelemay MJ, et al. Perfusion angiography of the foot in patient with critical limb ischemia: description of the technique. Cardiovasc Intervent Radiol 2015; 38: 201-5.

4) Hinrichs JB, Murray T, Akin M, et al. Evaluation of a novel 2D perfusion angiography technique independent of pump injections for assessment of interventional treatment of peripheral vascular disease. Int J Cardiovasc Imaging 2017; 33: 295-301.

5) Reekers JA, Koelemay MJ, Marquering HA, et al. Functional imaging of the foot with perfusion angiography in critical limb ischemia. Cardiovasc Intervent Radiol 2016; 39: 183-9.

6) Canty JM Jr, Judd RM, Brody AS, et al. First-pass entry of nonionic contrast agent into the myocardial extravascular space: effects on radiographic estimates of transit time and blood volume. Circulation 1991; 84: 2071-8. 